\title{
Maternal Health Care Providers and the Challenges of Quality Services in Rural Communities of Kaduna State, Nigeria

\author{
DOI: 10.36108/NJSA/2102/01(0140)
}

\author{
Godswill James \& Ada Helen Ochi \\ Department of Sociology \\ Ahmadu Bello University, Zaria
}

Vol. 10 Issue 1, 2012

\begin{abstract}
It is widely assumed by maternal health care programmers and policy makers that expanding coverage of maternal health care services will improve reproductive health indices such as maternal mortality; this is without taking into account the quality of care especially in rural areas. Despite various attempts by Governments to increase the utilization of maternity service and improve maternal health, the progress made so far remains little in rural areas. This study examines the challenges faced by maternal health care providers in providing quality services in rural communities of Kaduna State. A community-based cross-sectional research design was adopted and data were obtained using both quantitative and qualitative methods. A survey of 300 women aged 15-49 years was carried out, using multi-stage sampling techniques involving Local Government Areas, wards, main streets, houses, households and individuals. Focus group discussions were conducted among women who delivered three months before the survey, and those who experienced acute morbidity and complications. In-depth interviews were conducted with maternal health care providers in the study communities. The study reveals inadequacy in equipment, number and quality of staff in the rural health facilities; younger women were more likely to have positive experience of care than older women. Care was discontinued if health care providers were perceived as unqualified, neglectful or too young. Many pregnant women were discouraged from taking their drugs due to the perception that the drugs increased the baby's weight which makes delivery difficult. Engagement with political, religious leaders and community groups needs to be fostered through sensitization and sustained advocacy to remove constraints to accessibility of quality health care in rural areas.
\end{abstract}

Keywords: maternal health, pregnancy, maternal mortality, antenatal care, Kaduna State

\section{Introduction}

There is a growing assumption by maternal health care programmers that expanding coverage of reproductive health services would improve reproductive health indices such as maternal mortality; this is without taking into account the quality of care especially in rural areas. Quality health care service requires that necessary health related services are applied to safeguard mother and child health, and that user's satisfaction with care is achieved. However, the essential and enabling factors to guarantee this are limited or lacking in rural areas. The need for quality health care service is crucial as it is inextricably linked with maternal survival and health of the newborn. Due to 
the poor quality of health care received by pregnant women along the continuum of care, maternal and child health has worsened in Nigerian communities. Maternal mortality occurs largely due to delays and poor management of deliveries from cases that are avoidable such as prolonged labour, postpartum hemorrhage and infection from unhygienic handling of delivery.

To achieve the fifth millennium development goal for improved maternal health, the government has set in motion various policies and actions to enhance maternal survival. Despite the attempts by Government to reduce maternal mortality to the barest minimum, and to increase the utilization of maternity service and improved maternal health, the progress made so far is so little in rural areas as maternal mortality continues to remain high. For instance, in Nigeria, maternal mortality is among reproductive health problems, such as infant mortality and HIV/AIDS, that have adverse socio-economic consequences on individuals, the family, and communities, and in particular, impinges on overall level of progress and development of in the country. Nigeria Demographic and Health Survey (NDHS, 2008) estimated the national maternal mortality rate at 545 per 100,000 live births. Despite the apparent decrease, compared to previous rate of 800 per 100,000 live births in 2005, it is still high, and presents a picture of poor maternal health status of Nigeria. The government embarked on programmes to reduce maternal mortality such as, Midwife Service Scheme (MSS), aimed at achieving the fifth Millennium Development Goals (MDG) for increased maternal health and survival. However, the achievement made so far is low given that the annual percentage decline in maternal mortality ratio from 1990 to 2008 was $1.5 \%$ compared to the targeted 5.5\%. In addition, maternal deaths and lifetime risk is high as a woman's chance of dying from pregnancy and childbirth in Nigeria is 1 in 13, relative to 1 in 5000 in developed nations (UNICEF/WHO/UNFPA/ World Bank (2008); Federal Ministry of Health/National Primary Health Care Development Agency/Midwives Service Scheme (2009). To this effect, Nigeria's progress towards improving maternal health has been grossly inadequate.

A tudy by Idris et al. (2010) in Kaduna State, estimated maternal mortality ratio in three rural communities to be 1400 per 100,000 live births, a figure that is well above the national average of 545 per 100,000 live births. The final report document of the Partnership for Transforming Health Systems (PATHS, 2008) titled "Supporting Kaduna's Health Reform Agenda" revealed that mothers died frequently from complications of pregnancy and childbirth such as anaemia, obstetric hemorrhage, obstructed labour, sepsis and other reproductive health problems. The situation of health care service in the State showed that available resources were inadequate to support existing number of facilities and results in inadequate staffing of most facilities, poor maintenance of infrastructure, and insufficient supply of equipments, drugs and consumables. In addition, staff training was another key concern as some 
health workers operated without the requisite knowledge and skill base especially at primary health care level.

Obviously, the challenges of high maternal mortality lie with the quality rather than quantity of the health care provided (Zungu cited in Palitza, 2010). The structure put in place for maternal care and the process involved are interdependent since they influence the outcome of care rendered. The process and structures such as staffing level, skill, interpersonal relations, and equipment provision are essential because their adequacy or otherwise informs the quality of care received. Clearly, increased access to effective interventions and high quality of health care will improve maternal health and reduce mortality. Thus, this study investigates the challenges faced by maternal health care providers in providing quality services in rural communities of Kaduna State.

\section{Materials and Methods}

The study was conducted in three rural communities selected from each of the three Senatorial Districts in Kaduna State. The study was a community-based cross-sectional research, and the data collection instrument included questionnaires, focus group discussions and in-depth interview guides. A multi-stage sampling method was used in this research due to the vast area. The sampling was done in stages; at the first stage the population of Kaduna State was divided based on the existing three senatorial zones, each zone constituting a cluster. In the second stage, from each cluster, a Local Government Area from the list of Local Government Areas was selected. For the third stage, a ward was chosen from the selected local government areas. In Kaduna North Senatorial zone, Saka Dadi community was selected from Sabon Gari Local Government Area; in Kaduna Central, Giwa community was selected from Giwa Local Government Area; and from Kaduna South, Jaban Kogo community was selected from Kachia Local Government Area. Finally, a rural community was selected from the ward. This procedure was adopted in selecting three rural communities that constituted the study areas.

The main streets were randomly selected in each community, from which houses and households were systematically chosen to make up the sample for a particular area. In each household in the selected rural community, either a male or female was selected and if more than one male and one female, through a simple random sampling, a male and female respondents were selected. In all, 281 respondents (84 men and 197 women) were interviewed successfully, using structured questionnaires that elicited information on the respondent's socio-economic background; quality of health care service and women's experience of care; community perception of health care services rendered to women; challenges faced by maternal health care providers in the community; and how quality of health care service affects maternal mortality in the community. 
Two focus group discussions (FGDs) were conducted in each rural community, among women who delivered three months before the survey, and those who experienced acute morbidity and complications during their last pregnancy. The aim of the FGD was to elicit information on views of participants on actual quality of health care services, their experiences of care and how quality of health care service affects maternal mortality in their community. Their responses also provided possible suggestions to improve health care service in rural communities. FGD was adopted for the study because it provided more insight on health care service from service users in the community and thereby enriched data collected for the study. The composition of each group was fairly homogeneous with regard to age and marital status in order to minimize inhibitions in the flow of the discussions. Female bilingual indigenes were recruited and trained as moderators and note takers for the FGDs. A brief introduction to the study and purpose of involving participants was discussed, assuring anonymity and confidentiality; permission was also sought to tape-record the group discussion. The discussion in each group was conducted with the use of the FGD guide which contained lead questions, but flexibility was maintained in introducing the topics to ensure that participants are free to bring up issues they choose to discuss in response to particular questions. Participants were chosen in a non-probabilistic random fashion from various neighbourhoods or settlements to ensure a broad range of experiences within each group.

In addition, six in-depth interviews were conducted with maternal health care providers (Midwife/Traditional birth attendant) in the communities. This provided information on factual issues related to quality of health care women received, challenges in providing quality health care service and ways the quality of health care service affects maternal mortality. The interviews were conducted both in English and Hausa languages depending on the interviewees' choice. All the interviews were conducted in a very relaxed, unrushed atmosphere and relevant notes were taken by the interviewers.

\section{Results and Discussion}

\section{Assessment of Maternal Health Care Services}

The assessment relies on structures put in place for health care and the processes involved. Issues considered are affordability of charges, competence of care providers, outcome among pregnant women cared for, and adequacy of equipments. In addition, time devoted to pregnant women, number of staff, effectiveness of drugs supplied to pregnant women and their overall view of quality of health care services were also assessed. Tables 1 and 2 present the results for these. 
Table 1: Distribution of respondents by their responses on affordability of charges, outcome of pregnant women cared for, and competence of care provider

\begin{tabular}{|lrc|}
\hline Responses & Frequency & Percentage \\
\cline { 1 - 3 } Affordability of service charges & & \\
Very affordable & 43 & 15.3 \\
Affordable & 105 & 37.4 \\
Not affordable & 107 & 38.1 \\
No response & 26 & 9.3 \\
Total & $\mathbf{2 8 1}$ & $\mathbf{1 0 0 . 0}$ \\
& & \\
Competence of care provider in identifying & & \\
pregnancy related problem & & \\
Very competent & 41 & 14.6 \\
Competent & 161 & 57.3 \\
Not competent & 70 & 24.9 \\
No response & 9 & 3.2 \\
Total & $\mathbf{2 8 1}$ & $\mathbf{1 0 0 . 0}$ \\
& & \\
Outcome among pregnant women cared for by & & \\
care providers & 77 & 27.4 \\
Recover very well & 147 & 52.3 \\
Recover well & 50 & 17.8 \\
Do not recover well & 7 & 2.5 \\
No response & & \\
Total & $\mathbf{2 8 1}$ & $\mathbf{1 0 0 . 0}$ \\
\hline
\end{tabular}

Although slightly more than half of the respondents $(52.7 \%)$ said that service charges for pregnant women were affordable, the fact that more than one-third $(38.1 \%)$ said it was not affordable is worrisome. Service charges can discourage pregnant women in rural areas from seeking care even when available signs indicate that they require urgent medical attention. Regarding the competence of care providers, more than two-thirds $(71.9 \%)$ of the respondents were of the view that they are competent. However, evidence suggests that some care providers in health facilities in Kaduna did not possess requisite skills in maternal care. This was buttressed during FGDs with women who delivered three months prior to the survey, as summarized thus:

Sometimes when we bring our problems to our hospitals, the health workers will waste our time but in the end will not diagnose well, and the problem will persist. 
Similarly, a discussant from the group of women with complications in their last pregnancy summarized her feelings thus:

When I had my first delivery, I was taken to the hospital unconscious, spent more than six days there. I always attended ANC, was always examined during the visits but I still had the problem.

In addition, one of the TBAs further revealed that:

Some health workers do not know their work. There was a woman taken to Zabawa whom the health personnel told her baby was dead and that they will operate her. I decided to examine her myself and noticed that the baby was alive, and I told the mother. After the operation the baby was alive.

Data on the outcome of pregnant women cared for show that a vast majority of them $(79.7 \%)$ recover well compared to $17.8 \%$ who did not recover well. Pregnancy complications and poor recovery after birth generally drew community resentment and discouragement for hospital-based care. This is captured by a discussant in the FGD with women who had complications in their last pregnancy:

Some women in the community mock us who have complications and poor recovery; they say to us, you people always go for ANC yet you develop problems, but look at us that don't go, Allah helps us to give birth safely at home.

Such statements give an insight on how poor pregnancy management and delivery outcome can influence negative response or attitudes towards modern pregnancy care and utilization of maternal health care services. This is even much more aggravated if there is perceived incompetence among health workers and high cost of services.

Table 2 shows that the majority of the respondents (54.4\%) are of the view that equipments for detecting pregnancy-related conditions or diseases are not adequate in health facilities in rural communities of Kaduna State. This implies that most cases that require further tests, scanning and screening are undertaken outside the rural vicinity at best, or may be ignored completely with obvious consequences. 
Table 2: Distribution of respondents by their responses on adequacy of equipment, number of staff, effectiveness of drugs supply and quality of health care service

\begin{tabular}{|c|c|c|}
\hline Responses & Frequency & Percentage \\
\hline \multicolumn{3}{|c|}{$\begin{array}{l}\text { Adequacy of equipment for detecting pregnancy } \\
\text { related disease }\end{array}$} \\
\hline Very adequate & 13 & 4.6 \\
\hline Adequate & 107 & 38.1 \\
\hline Not adequate & 153 & 54.4 \\
\hline No response & 8 & 2.8 \\
\hline Total & 281 & 100.0 \\
\hline \multicolumn{3}{|l|}{ Number of staff } \\
\hline Very adequate & 13 & 4.6 \\
\hline Adequate & 103 & 36.7 \\
\hline Not adequate & 157 & 55.9 \\
\hline No response & 8 & 2.8 \\
\hline Total & 281 & 100.0 \\
\hline \multicolumn{3}{|c|}{$\begin{array}{l}\text { Effectiveness of drugs supplied to pregnant } \\
\text { women }\end{array}$} \\
\hline Very effective & 96 & 34.2 \\
\hline Effective & 116 & 41.3 \\
\hline Not effective & 62 & 22.1 \\
\hline No response & 7 & 2.5 \\
\hline Total & 281 & 100.0 \\
\hline \multicolumn{3}{|c|}{ Quality of care pregnant women received } \\
\hline Very good & 18 & 6.4 \\
\hline Good & 128 & 45.6 \\
\hline Poor & 113 & 40.2 \\
\hline Very poor & 16 & 5.7 \\
\hline No response & 6 & 2.1 \\
\hline Total & 281 & 100.0 \\
\hline
\end{tabular}

Similarly, the majority of the respondents consider the number of staff inadequate in relation to the clients. This may have implications for waiting time or delay in receiving treatment. In addition, the quality of care is adjudged to be poor by a significant number of the respondents (about 46 percent). However, care providers tend to be rated high in terms of the effectiveness of drugs supplied. However, revealing reports from FGDs indicate that despite the favourable perception regarding the effectiveness of drugs supplied, their 
utilization seems to be shrouded in misconceptions and fears. Two participants captured the general feelings of the group thus:

Some will say, do not take the yellow tablet or the red one. People in the community will say if you drink those drugs, the baby will be very large, and when you cannot deliver, this will make us take the woman to them in the hospital.

Women always complain that the drugs given at ANC make the baby grow too big and when it comes to delivery, it is very difficult, so some will throw away the medicine.

These responses are indications of ignorance and misinformation as a crucial challenge to the attainment of maternal health in rural areas, and ultimately suggest a need to design educational programs or strategies, which will promote a new consciousness and improve attitudes towards all components of maternal health care. Generally, the negative perception of the effectiveness of drugs supplied to pregnant women or their non utilization implies that the anticipated vital role of these drugs may not be achieved among pregnant women, and this may worsen the maternal health status in rural areas.

\section{Challenges of Maternal Health Care Providers}

To find out what serves as major challenge of maternal health care providers in the community, questions were directed to respondents to identify from an option of variables on drugs and equipment, skill and motivation and distance. In addition, challenge was assessed in terms of differences encountered when maternal care providers relate to pregnant women. Furthermore, the facility health checklist administered to maternal health care providers addressed availability of skilled staff and essential materials, assessed accessibility to health care service in terms of cost and 24 hour service, and component of antenatal care service received as well as client-provider interaction, an indicator in the process of care that constitutes women's experience of care. The responses are presented in this section including suggestions on how challenges can be improved (Table 3). 
Table 3: Responses on the major challenges of maternal health care providers

\begin{tabular}{|lrrr|}
\hline Responses & & Frequency & Percentage \\
\cline { 1 - 4 } $\begin{array}{l}\text { Major challenge faced by } \\
\text { providers }\end{array}$ & & \\
Lack of routine drugs and equipments & 32 & 55.9 \\
Poor staff motivation & 24 & 11.4 \\
Distance to work place & 50 & 8.5 \\
Inadequacy of skilled manpower & 1 & 17.8 \\
No challenge & 17 & 0.4 \\
No response & & 6.0 \\
Total & $\mathbf{2 8 1}$ & $\mathbf{1 0 0 . 0}$ \\
\hline
\end{tabular}

The above distribution indicates that a large majority of respondents $(55.9 \%)$ consider lack of routine drugs and equipments as the major challenge care givers encounter, followed by inadequacy of skilled manpower. A Midwife, one of the key informants, provided some insights into the problem:

Not all equipments for delivery are available, for instance, the forceps. We just keep managing things, and we don't have enough. Government officials will come and make promises that they will repair and equip the hospital, recruit and post more staff to us but you will not see them. So, if there are no drugs or equipments, how can we do our work well?

This suggests that lack of physical resources is a major challenge of care providers to carry out their duties effectively and government's lack of commitment is the root cause of the problem. Furthermore, the respondents were asked to identify differences that exist between them and care givers that could serve as a challenge. Their responses show that educational difference $(29 \%)$ and gender differences $(24 \%)$ were the two most important differences, followed by differences in socio-economic status (19.2\%) and language difference $(18.5 \%)$. This implies that educational and gender differences are the most common challenges when care providers relate with pregnant women. The former hinders effective communication while the latter results from nonavailability of female health care providers, especially doctors, in rural areas. Importantly, maternal health care providers shed light on their challenges; the various situations were presented as follows:

We do not run shift in the clinic so I answer midnight calls to attend to pregnant women. The local government does not pay me anything 
apart from my salary. This is why I feel discouraged at times and I don't answer some calls.

Sometimes, a woman will be in labour and tell you, she came for antenatal. When the history of the pregnancy is taken and the edge is measured, you will realize that she is in labour. Just the first visit.

My problem is that some women don't like attending ANC and when I can't handle their delivery and take them to the hospital, I am the one that receives the blame as they will say I don't use to tell them to come for $A N C$.

Those in their first pregnancy like the Fulanis are shy, and when they come to the hospital and you ask her questions about the pregnancy and even her name, she will not answer. Gradually and patiently you will try to get information from the mother in-law whom she came with. It can be very frustrating sometimes!

Sometimes the women do not come on time for ANC, they come almost closing hour around 2 o'clock pm.

These responses suggest that the challenges encountered in the relationship between pregnant women and care providers vary; however, it can be summed up as lack of motivation, shyness of primigravida, lateness and non-attendance of antenatal care. The implications of these challenges to maternal care providers are in various ways. For instance, there are challenges in the working environment such as lack of equipments, drugs and motivation frustrate care givers, which in turn lead to poor attitudes pregnant women. The second aspect is the action of the pregnant women, when they do not attend antenatal care and present their cases late to the hospital. This will not give care providers adequate time to undertake basic checks and when they arrive with complication, assembling necessary drugs/equipments that would improve maternal health outcome would be delayed. 
Table 4: Human, physical, and essential materials available at PHCs

\begin{tabular}{|lllll|}
\hline Human, physical and essential materials & Giwa & & Saka Dadi & $\begin{array}{l}\text { Jaban } \\
\text { Kogo }\end{array}$ \\
\cline { 5 - 5 } \cline { 4 - 5 } Number of midwife & & 1 & 0 \\
Dork full time & Yes & No & No \\
Number of delivery beds & No & No & No \\
Laboratory & 3 & 2 & 1 \\
Partograph chart & Yes & Yes & No \\
Stethoscope & Yes & No & No \\
Adult weighing scale & Yes & Yes & Yes \\
Thermometer & Yes & Yes & Yes \\
Blood pressure machine/cuff & Yes & Yes & Yes \\
Latex glove & Yes & Yes & Yes \\
Ambulance/emergency tran sport & Yes & Yes & Yes \\
Infusion kit for intravenous solution & No & No & No \\
Autoclave for sterilization & Yes & Yes & No \\
Water supply/electricity & Yes & No & No \\
Environmental disinfectant available always & Yes & Yes & No \\
Magnesium sulphate available always & Yes & Yes & Yes \\
Oxytocin injection available always & Yes & Yes & Yes \\
\hline
\end{tabular}

With reference to skilled maternal health care provider, Table 4 above reveals that medical doctors are not available, midwives are inadequate, and the chances of being attended to by a midwife at any point in time is low, as not all work full time. Laboratory, partograph chart, infusion kit, and autoclave for sterilization of surgical instruments were not available in two health centres. The implication of this state of resources is that it contributes to low quality of care. Although, environmental disinfectant, thermometer, adult weighing scale, stethoscope were available in all the health centres, two essential drugs, oxytocin and magnesium sulphate, which are crucial for saving maternal life during emergency in the management of haemorrhage and eclampsia were always available in the health centres except the one at Jaban Kogo, where magnesium sulphate was not always available. This suggests that women presented with cases of eclampsia may not receive the urgent medication needed to prevent adverse outcome.

Regarding availability of emergency transport, none of the health centres has ambulance/emergency transport, an indicator in the process of maternal care for ensuring timely care for referred complicated cases. The key informants in all the three communities disclosed that in circumstances of non availability of ambulance during cases of emergency, the pregnant woman cannot but use a motorcycle as means of transport, and if she did not have money, nothing can be done. In most cases, when a referral letter is given, the 
pregnant woman would go on her own; the private car owners sometimes assist, however, motorcycle is mostly used as an alternative means for emergency transportation.

Essentially, the poor enabling environment and lack of motivation among health workers adversely affect their performance in rendering services. According to the Kaduna State Government (2011), poor conditions of service, including inadequate staff housing, have made it extremely difficult to recruit and retain staff particularly in rural areas. Murray and Frenk (2000) were of the opinion that gap in knowledge and inappropriate applications of available technology could be a challenge and results to low quality of care. There are some underlying factors which may produce a low standard of care for the patient. This includes situations produced by the action of the woman herself or her relatives, which may be outside the control of the clinician. It also takes into account shortages of resources for staffing facilities, administrative failure in maternity service, and the back-up facilities such as anesthetics services (Victorian Government, 2001).

Among rural communities, social distance serves as a barrier to access services. Social distance consists of differences in language, behaviour and expectations between the consumer of health care and its providers. Ethnic and linguistic differences, and even when providers are of the same ethnic group, there can be social distance barriers caused by differences in education, experience, and socioeconomic status (PNMN, 1997 cited in Ladipo, 2008).

The distribution of health infrastructures favours urban at the expense of rural areas. The lopsided distribution affects the quality of health care services available and accessible in rural areas where maternal health problems are common. In times of emergency, the existing poor health care service in rural areas becomes highly ineffective to save life as skilled personnel, life saving equipments, essential drugs and ambulance are either unavailable or inadequate. The implications of these problems are that it will affect the level of acceptability and uptake of maternity service which focus is to reduce maternal mortality. Secondly, women's experience of care will strongly influence her future health-seeking behaviour. Finally, efforts to reduce maternal mortality in rural areas from the dimension of health care service may not be achieved as targeted.

\section{Conclusion}

Indeed, maternal health challenges are common in rural areas where the population is largely poor, less educated, and less well-informed on maternal health; they are less likely to have access to trained medical personnel, and less likely to have equipped health centres, due to inequality in distribution of health infrastructures at the disadvantage of rural areas. This study has revealed that the quality of health care, comprising structures and facilities put in place and care delivery, are a far cry from what is imperative to improve maternal 
health outcome and reduce maternal mortality in rural areas. Inevitably, maternal health will improve considerably in rural communities when there are greater investments in human resources, infrastructures for maternal health and proper attention to women's experience in the process of care.

Innovative engagement with political, religious leaders and community groups needs to be fostered through sensitization and sustained advocacy to remove constraints to accessibility of quality health care in rural areas in Kaduna State. This may also include greater engagement of civil society organization and the private sector.

\section{References}

Federal Ministry of Health and National Primary Health Care Development Agency (NPHCDA) Midwives Service Scheme. 2009. Accelerating Reduction in Maternal, Newborn and Child Mortality through Improved Access to Skilled Birth Attendants Pp 3, 5. Retrieved 25 May, 2011 www.nphcda.gov ng/.../MSS_HARMONIZED_CONCEPT_FINAL_ATREIZ-HTL.doc

Idris, H.; Tyoden, C.; Ejembi, C. \& Taylor, K. 2010. Estimation of Maternal Mortality Using the Indirect Sisterhood Method in Three Communities in Kaduna State, Northern Nigeria. African Journal of Reproductive Health. 14(3):79.

Kaduna State Government. 2011. Overview of Kaduna State. Retrieved May 30, 2011 from www.Paths2.org/index.php.

Ladipo, O.A. 2008. Delivery of an Effective Maternal and Child Health Services in Nigeria. Retrieved May 20, 2011 from www.ngnhc.org/.../ delivery\%20of $20 \%$ effective\%20materna.

Murray, C.J. \& J. Frenk. 2000. A Framework for Assessing the Performance of Health Systems. Bulletin of the World Health Organization. 78 (6): 71731.

National Population Commission (NPC) [Nigeria] and ICF Macro. 2009. Nigeria Demographic and Health Survey 2008. Abuja, Nigeria: National Population Commission and ICF Macro.

Partnership for Transforming Health System. PATHS, 2008. Supporting Kaduna's Health Reform Agenda. Retrieved August 30, 2011 from http://www.healthpartners.int.co.uk/our_project/document/kadunapathsrep ort.

Palitza, K. 2010. Africa Lack of Quality Health Care Causes Rise in Orphans. Retrieved March 30, 2011 from www.ips.news.net.

UNICEF/WHO/UNFPA/World Bank. 2008. Trends in Maternal Mortality. Retrieved March 15, 2011 from www.whqlibdoc.who.int/ publications/2010. 
150 The Nigerian Journal of Sociology and Anthropology Vol. 10

Victorian Government. 2001. Measuring Maternity Care: A Set of Performance Indicator. Retrieved May 30, 2011 from http://www.dhs.vic.gov.au/ahs/ quality/effect.htm. 